\title{
Automated fiducial point selection for reducing registration error in the co-localisation of left atrium electroanatomic and imaging data
}

\author{
Rheeda L Ali ${ }^{1}$, Chris D Cantwell ${ }^{2}$, Norman A Qureshi ${ }^{3}$, Caroline H Roney ${ }^{1}$, \\ Phang Boon Lim $^{3}$, Spencer J Sherwin ${ }^{2}$, Jennifer H Siggers ${ }^{1}$, Nicholas S Peters ${ }^{3}$
}

\begin{abstract}
Registration of electroanatomic surfaces and segmented images for the co-localisation of structural and functional data typically requires the manual selection of fiducial points, which are used to initialise automated surface registration. The identification of equivalent points on geometric features by the human eye is heavily subjective, and error in their selection may lead to distortion of the transformed surface and subsequently limit the accuracy of data co-localisation. We propose that the manual trimming of the pulmonary veins through the region of greatest geometrical curvature, coupled with an automated angle-based fiducial-point selection algorithm, significantly reduces target registration error compared with direct manual selection of fiducial points.
\end{abstract}

\section{INTRODUCTION}

Patient-specific modelling of cardiac electrophysiology, integrating both clinical intracardiac mapping and imaging modalities, has the potential to inform clinical intervention in cases of complex cardiac rhythm disturbances, such as atrial fibrillation. To accurately predict cardiac conduction in this context requires the precise co-registration of the available patient data to effectively integrate into a computer model.

Electroanatomic (EA) mapping, where intracardiac electrodes record electrical and positional information when placed in contact with the endocardium, are routinely collected during investigative clinical procedures. These data are subsequently interpreted through summary statistics, such as local activation time (LAT) or measures of fractionation, to assist in the diagnosis of the condition. The annealed positional information allows a reconstruction of the chamber geometry onto which the data is displayed. In contrast, magnetic resonance imaging (MRI), in combination with a contrast enhancement agent, delineates anatomical structure. Delayed imaging allows for a reduction of the contrast agent in healthy myocardium, thereby enabling the identification of diseased tissue, represented by high intensity voxels in the image.

Since the electrical data and the scar delineation lie in different coordinate systems, the electrical data is often trans-

\footnotetext{
${ }^{1}$ Department of Bioengineering, Imperial College London, South Kensington Campus, London SW7 2AZ, UK $\{$ rheeda.ali07, caroline.roney10, j.siggers $\}$ at imperial.ac.uk

${ }^{2}$ Department of Aeronautics, Imperial College London, South Kensington Campus, London SW7 2AZ, UK $\{$ c. cantwell, s. sherwin $\}$ at imperial.ac.uk

${ }^{2}$ National Heart and Lung Institute, Imperial College, 4th floor, Imperial Centre for Translational and Experimental Medicine, Hammersmith Campus, Du Cane Road, London W12 0NN, UK \{n.qureshi, p.b.lim, n.peters\} at imperial.ac.uk
}

formed (registered) onto the late gadolinium enhanced (LGE) MRI surface. Typically, the EA surface is first registered with the LGE-MRI, and the resulting transformation is then applied to map the electrical data onto the LGE-MRI surface.

Landmark registration provides a first estimate for surface registration and is usually based on the manual selection of corresponding fiducial points (landmarks) on the two surfaces (or images) which are to be registered [1]. Sra et al. investigated landmark registration between canine left atrial geometries, obtained from computer tomography (CT) and the EnSite electroantomical mapping system. Prior to CT scanning, fiducial points were first manually inserted into the LA chamber as pacing leads [2]. The CT surface was then exported into the electro-anatomical system, where a catheter was used to choose the corresponding fiducial points. Using these landmarks, the mean registration error was estimated as $2.0 \pm 3.6 \mathrm{~mm}$. Fahmy et al. observed that the best landmark registration was obtained when the fiducial points were selected on the pulmonary veins (PVs) $(5.6 \pm 3.2 \mathrm{~mm})$, but higher registration errors were obtained with fiducials chosen on the coronary sinus (CS) and left atrial appendage (LAA) [3].

In previous work, landmarks were manually selected on both the LGE-MRI and EA surface and landmark registration was then performed using a series of rigid, affine and non-rigid transformation within the Image Registration Toolkit software[4], [5], [6], [7]. However, manual landmark selection can be highly subjective, since it is dependent on the electrophysiologist choosing anatomically corresponding points. As such, we propose an automated method of landmark selection, where points are chosen only on the PVs of both geometries, and compared it with the existing manual approach.

\section{METHODS}

\section{A. Manual Landmark Selection}

The LGE and EA left atrial surfaces were displayed using a custom-written rendering platform, from which an experienced electrophysiologist manually selected anatomically corresponding points (numbering approximately 16) on each geometry. The points were primarily selected around clear anatomical features, such as PVs ostium and carina, as illustrated in Figure 1, which shows example EA and LGEMRI geometries with corresponding landmarks selected . 
(a)

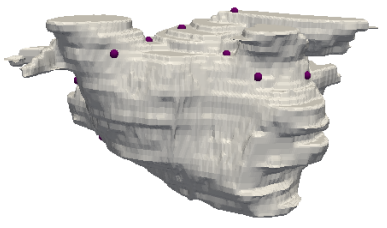

(b)

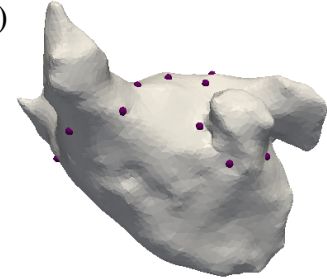

Fig. 1: Example of manually selected landmarks by an experienced electrophysiologist on (a) the LGE-MRI surface, and (b) the electroanatomic surface.

\section{B. Automatic Landmark Selection}

The LGE-MRI image was imported into ITK-snap [8], and manually segmented by selecting the blood pool within the atrial structures including the PVs, the LAA and the mitral valve (MV) to create a triangulated surface of the endocardium (the LGE-MRI surface). The left atrial endocardial geometry (EA surface) was exported from the electroanatomical mapping system. A $180^{\circ}$ rotation around the $z$ axis was applied to the EA surface to orient it correctly with the LGE-MRI surface. The triangulated surfaces were imported into the mesh manipulation package Blender [9] for pre-processing, where the PVs were trimmed to the body of the atrium. Each cut was defined as the plane intersecting the points of maximum curvature in the PV-atrial junction. An edge detection algorithm was applied to each processed surface to extract a sequence of line segments defining the PV boundaries.

For each vein, the centre point of the LGE-MRI boundary and the corresponding EA boundary were computed and oriented with outward-facing normal, computed from the centre of mass of the surface. The boundary co-ordinates of each PV were projected onto a two-dimensional plane of best fit and the centre points aligned. Landmarks were automatically selected on the PV boundaries. Different distributions of landmarks, and their impact on the registration process, were considered.

The number of generated landmarks $n$ per PV was chosen within the range $2 \leq n \leq 8$. A corresponding number of rays, distributed around the origin of the plane at equal angle, were intersected with the projected vein boundaries to determine the landmark points. The initial landmark was chosen at an anti-clockwise offset $\theta$ from the $x$-axis, where $0 \leq \theta<2 \pi / n$. Identical offsets were applied to both the LGE and EA landmarks. The corresponding point on the original three-dimensional geometry This process was repeated for the EA surface using the same value of $n$. In assessing the impact of $\theta$ on the resulting registration, points were evaluated at 1-degree intervals.

To assess the effect of the imposed equidistant criterion, landmark points were also selected based on random angles from 0 to $2 \pi$. Identical landmark distributions were chosen on each of the LGE and EA geometries.

(a)

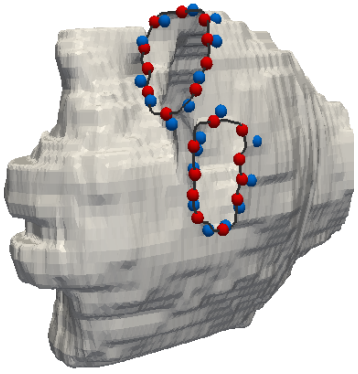

(b)

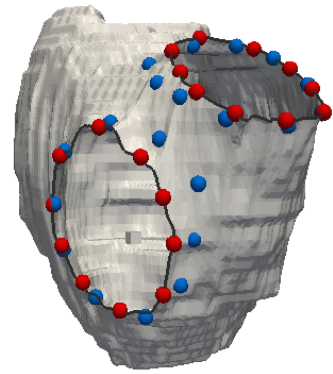

Fig. 2: Automated landmark selection (red) and the corresponding transformed landmarks on four veins, shown on (a) left posterior view (b) right posterior view.

\section{Target Registration Error}

Registration is performed using IRTK [5], [6], [7]. Initially, a rigid landmark registration is performed using the landmarks selected either manually or through the automated procedure. Further non-rigid and affine registrations were performed to refine the landmark transformation. The resulting transform is used as an initial condition for a surface registration between the LGE-MRI and EA pre-processed surfaces.

Unlike Fiducial Registration Error (FRE), which measures the error in the registration of the landmark points themselves, Target Registration Error (TRE) is defined as the Euclidean distance between non-fiducial points $\mathbf{y}$ and the transformation $\mathcal{T}(\mathbf{x})$ of their corresponding points $\mathbf{x}$ on the other surface [10], that is,

$$
\operatorname{TRE}(\mathbf{x})=\mathcal{T}(\mathbf{x})-\mathbf{y} .
$$

To compute this, ten equidistant points, offset to avoid coincidence with fiducial points, were generated on each PV and on each surface, resulting in a total of forty points on each surface. The chosen offset was the same on both surfaces. The value $\overline{\mathrm{TRE}}$ is defined as an average TRE across possible landmark offsets.

\section{RESULTS}

In this section we examine the quality of the registration produced by using the automated landmark selection process, using left atrial geometries from eight patients. In this study we specifically vary the number of landmarks, their offset from the $x$-axis and their distribution.

\section{A. Dependence on number of landmarks}

For each value of $n$, the registration error was sampled at angular shifts, regularly spaced $1^{\circ}$ apart, and the $\overline{T R E}$ was calculated for each $n$. The solid lines in Fig. 3 shows the TRE for each $n$, for all eight data sets. With increasing $n$, there is a slight overall trend towards lower $\overline{\mathrm{TRE}}$. Of the 8 data sets, 5 were found to have the lowest TRE for $n \geq 6$. For the remaining datasets, little change in TRE was observed with $n$, indicating that a small number of landmarks per vein was sufficient to initialise the surface registration process. 


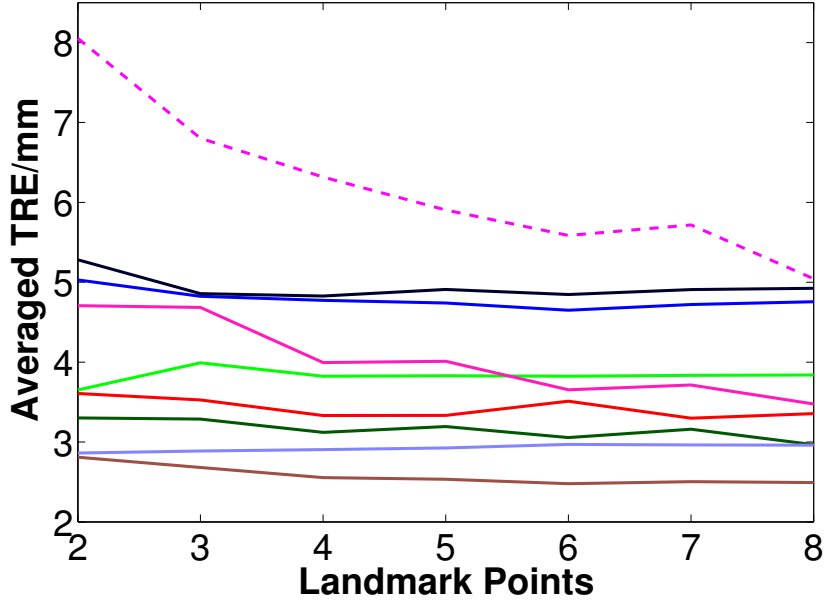

Fig. 3: Calculated $\overline{\mathrm{TRE}}$ for increasing number of landmarks per vein in 8 datasets. The dotted line shows the $\overline{\mathrm{TRE}}$ for in the case of randomly selecting landmarks.

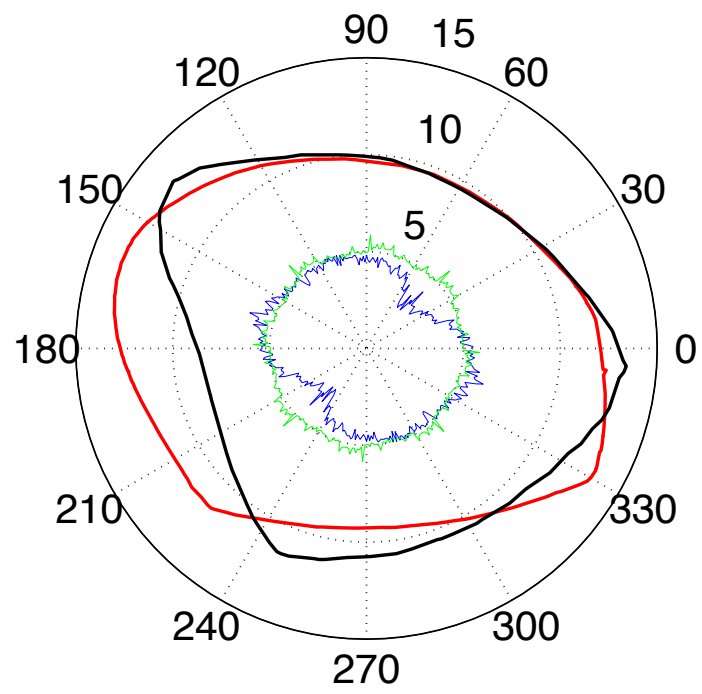

Fig. 4: Polar plot of TRE as a function of angular shift $\theta$ (blue) for a single vein with $n=2$ (blue) and $n=8$ (green) landmarks. The vein boundaries of the MRI (red) and EA (black) geometries are superimposed.

\section{B. Dependence of landmark position}

Registration errors were computed for equally spaced landmarks rotationally shifted by an angle $0 \leq \theta<2 \pi / n$, for each value of $n$. For the case where $\overline{\mathrm{TRE}}$ is lowest at $n=2$ (green line in Fig. 3), Fig. 4 compares the TRE distribution for $n=2$ and $n=8$ for a single vein. While there is clearly little variation with $\theta$ in the magnitude of TRE at $n=8$, the TRE at $n=2$ shows a significant reduction in error at approximately $\theta=45^{\circ}$.

It was also observed that the TRE, computed per vein, sometimes varied substantially with $\theta$. A second example of a TRE distribution for a single vein is shown in Fig. 5. Lower TRE was observed where the vein boundaries were mostly parallel to each other, with higher error occuring in the grey

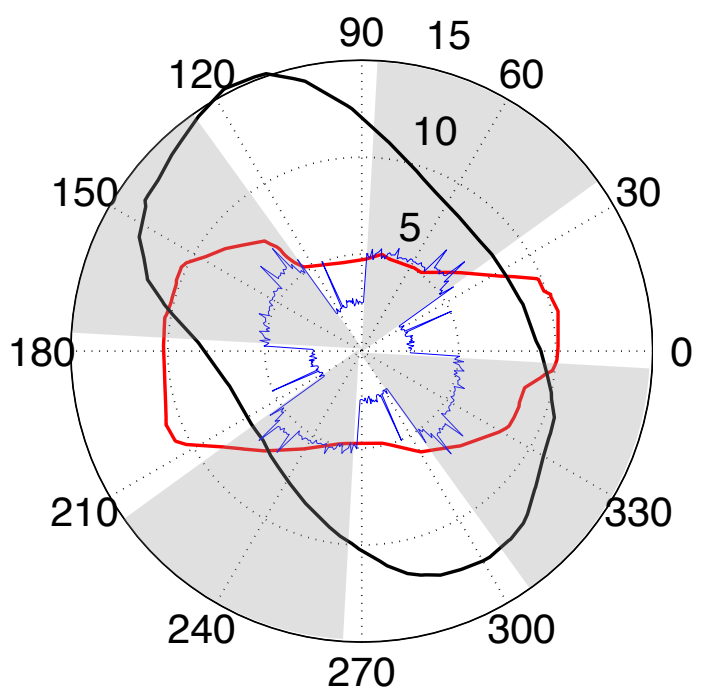

Fig. 5: Polar plot of TRE as a function of angular shift $\theta$ (blue) for a single vein with $n=4$ landmarks. The vein boundaries of the MRI (red) and EA (black) geometries are superimposed. Grey areas denote the regions of higher TRE.

regions.

It was further observed that the TRE, plotted as a function of $\theta$, differed significantly between veins within the same dataset. Fig. 6 compares the TRE and boundaries of each vein, for the LGE-MRI and EA surfaces, for two datasets with the $\overline{\mathrm{TRE}}$ for $n=4$. Error was frequently higher when landmarks were placed in sections of the vein boundaries which were locally deformed, suggesting that this introduced distortion during the registration process which negatively impacted the co-location of the non-fiducial points.

\section{Landmark distribution}

We compared the equally spaced landmarks with randomly positioned landmarks, for each value of $n$, to investigate the importance of the distribution. The $\overline{\mathrm{TRE}}$ was consistently higher, for all values of $n$, for randomly selected landmarks, an example of which is shown by the dotted purple line in Fig. 3, in comparison with the solid purple line obtained using the automated technique. This suggests that the equidistant angle criterion, used in our semi-automated approach, is important in generating accurate registration.

\section{Comparison with manual selection}

At the electrophysiologists discretion, approximately 16 landmarks were typically chosen during the manual landmark selection. This corresponds to $n=4$ landmarks in the automated algorithm. Selection was made on the unprocessed surface, an example of which is shown in Fig. 1. The same set of non-fiducial points were used to compute the TRE in both cases. The manual landmark selection produced a TRE which was always higher than that of the automated approach $(\delta=2.22 \pm 1.42 \mathrm{~mm})$. 

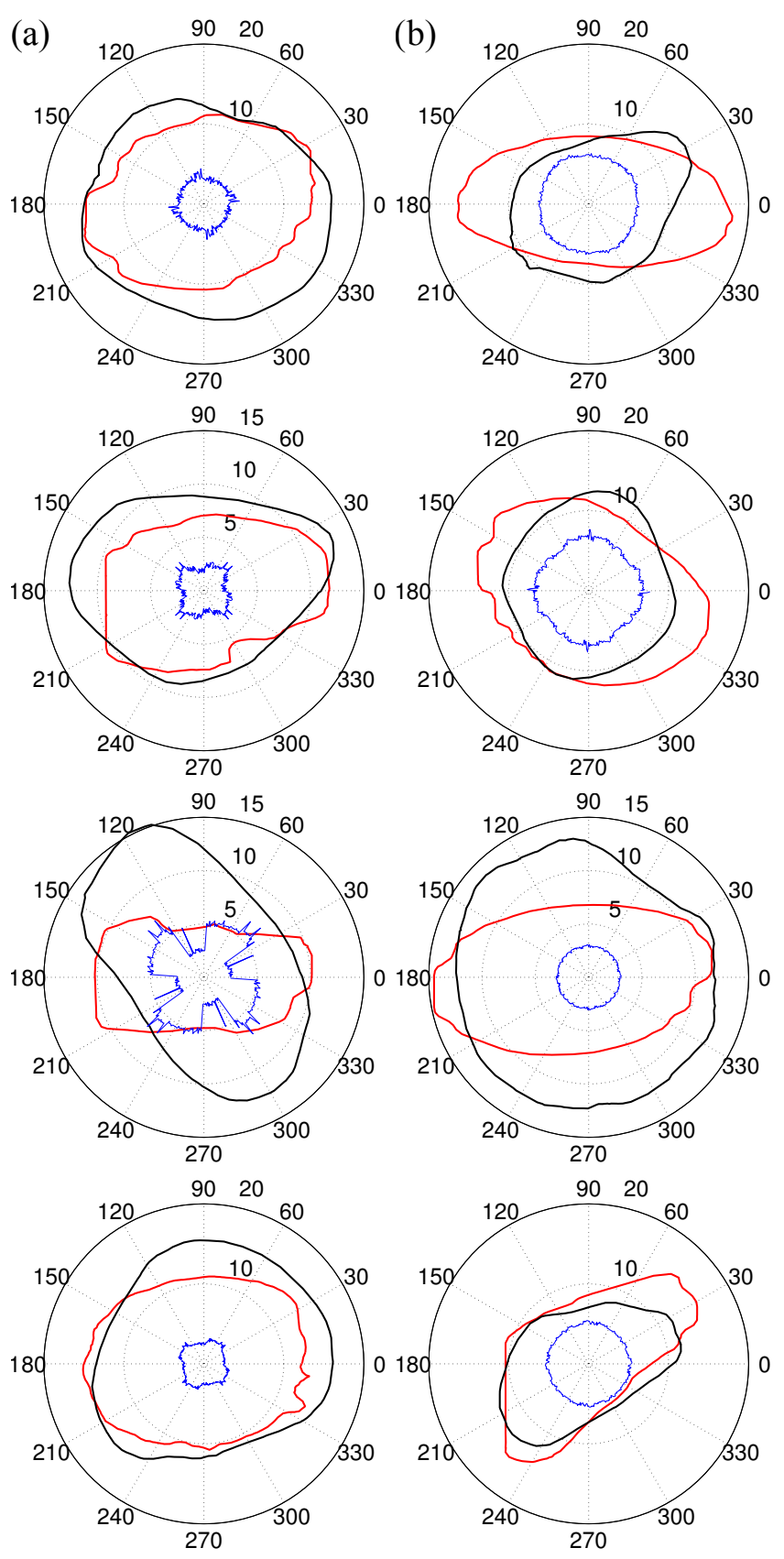

Fig. 6: TRE distribution (blue) for the four PVs for two datasets (a) and (b) where $n=4$. Vein boundaries from the MRI (red) and EA (black) surfaces are shown.

\section{DISCUSSION}

This work aimed to develop a semi-automated approach to registration between surfaces generated from MRI and electro-anatomic mapping systems. Pulmonary vein boundaries were projected onto a plane of best fit and fiducial points were placed at equal angle. We investigated the impact on the target registration error of the number, distribution and positioning of landmark points using eight datasets.

Increasing the number of landmarks was found to slightly improve registration on average, although some datasets showed little improvement with higher numbers of land- marks. The $\overline{\mathrm{TRE}}$ varied substantially across the veins of an individual dataset, where dissimilar boundaries produced higher $\overline{\mathrm{TRE}}$ values. Furthermore, TRE varied with $\theta$ within an individual vein and the variation could be accounted for by localised differences in the vein morphology. Equal distribution of landmarks provided a better registration than choosing the landmarks randomly.

One limitation of the method is that during segmentation the left atrial appendage and the left inferior pulmonary vein were often in close proximity, which made accurate cutting of the PVs in the plane of highest curvature challenging. Due to the difficulty in positioning corresponding points on the atrial walls of both surfaces, the registration in the main body of the atrium could not be assessed. However, previous studies [3] have reported that the pulmonary veins offer the most reliable locations for placing fiducial points. In the future the landmark selection could be completely automated to select the optimal placement of fiducial points.

\section{ACKNOWLEDGMENT}

This work was supported by the British Heart Foundation (BHF), grants FS/11/22/28745 \& RG/10/11/28457, the ElectroCardioMaths Programme of the Imperial BHF Centre of Research Excellence and NIHR Imperial Biomedical Research Centre.

\section{REFERENCES}

[1] J. Sra and S. Ratnakumar, "Cardiac image registration of the left atrium and pulmonary veins," Heart Rhythm, vol. 5, no. 4, pp. 609-617, 2008.

[2] J. Sra, D. Krum, J. Hare, D. Okerlund, H. Thompson, M. Vass, J. Schweitzer, E. Olson, W. D. Foley, and M. Akhtar, "Feasibility and validation of registration of three-dimensional left atrial models derived from computed tomography with a noncontact cardiac mapping system," Heart Rhythm, vol. 2, pp. 55-63, Jan. 2005.

[3] T. S. Fahmy, H. Mlcochova, O. M. Wazni, D. Patel, R. Cihak, M. Kanj, S. Beheiry, J. D. Burkhardt, T. Dresing, S. Hao, P. Tchou, J. Kautzner, R. A. Schweikert, M. Arruda, W. Saliba, and A. Natale, "Intracardiac echo-guided image integration: Optimizing strategies for registration,' Journal of Cardiovascular Electrophysiology, vol. 18, pp. 276-282, 2007.

[4] R. Ali, C. Cantwell, C. Roney, N. Qureshi, P. Lim, J. Siggers, S. Sherwin, and N. Peters, "A novel method for quantifying localised correlation of late-gadolinium intensity with conduction velocity," in Computing in Cardiology, (Boston, MA), pp. 193-196, 2014.

[5] D. H. C. Studholme, D.L.G.Hill, "An overlap invariant entropy measure of 3D medical image alignment," Pattern Recognition, vol. 32, no. 1, pp. 71-86, 1999.

[6] D. Rueckert, L. I. Sonoda, C. Hayes, D. L. Hill, M. O. Leach, and D. J. Hawkes, "Nonrigid registration using free-form deformations: application to breast MR images.," IEEE Transactions on Medical Imaging, vol. 18, no. 8, pp. 712-721, 1999.

[7] J. Schnabel, D. Rueckert, M. Quist, J. M. Blackall, A. D. S. Castellano, T. Hartkens, G. P. Penney, W. A. Hall, H. Liu, W. A. Truwit, F. A. Gerritsen, D. L. G. Hill, and D. J. Hawkes, "A Generic Framework for Non-rigid Registration Based on Non-uniform Multi-level Free-Form Deformations - Springer," Fourth Int. Conf. on Medical Image Computing and Computer- Assisted Intervention (MICCAI '01), p. 573, 2001.

[8] P. A. Yushkevich, J. Piven, H. Cody Hazlett, R. Gimpel Smith, S. Ho, J. C. Gee, and G. Gerig, "User-guided 3D active contour segmentation of anatomical structures: Significantly improved efficiency and reliability," Neuroimage, vol. 31, no. 3, pp. 1116-1128, 2006.

[9] R Core Team, R: A Language and Environment for Statistical Computing. R Foundation for Statistical Computing, Vienna, Austria, 2013.

[10] J. M. Fitzpatrick and J. B. West, "The Distribution of Target Registration Error in Rigid-Body Point-Based Registration," IEEE TRANSACTIONS ON MEDICAL IMAGING, vol. 20, no. 9, pp. 917-927, 2001. 